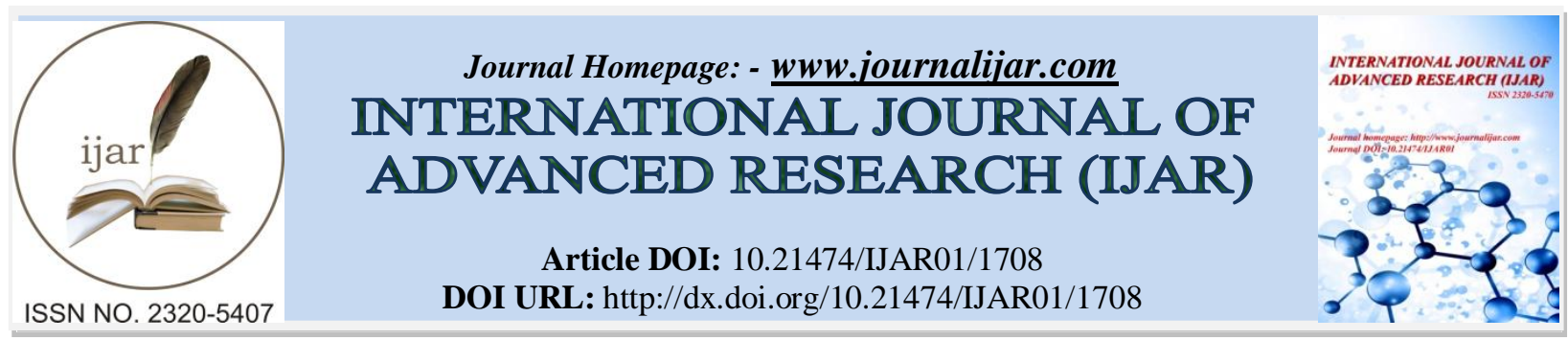

RESEARCH ARTICLE

\title{
SYSTEMATIC SIGNIFICANCE OF FOLIAR CHARACTERS IN THE DELIMITATION OF PASSIFLORA L. (PASSIFLORACEAE) AT THE LOWER TAXONOMIC LEVELS.
}

${ }^{*}$ VL Beena ${ }^{1}$ and S Suhara Beevy ${ }^{2}$.

1. Research Scholar, Department of Botany, University of Kerala, Kariavattom, Thiruvananthapuram, Kerala, India.

2. Assistant Professor, Department of Botany, University of Kerala, Kariavattom, Thiruvananthapuram, Kerala, India.

\section{Manuscript Info}

\section{Manuscript History}

Received: 12 July 2016

Final Accepted: 19 August 2016

Published: September 2016

\section{Key words:-}

Passiflora; foliar morphology; ANOVA; intra specific diversity; inter specific diversity

\section{Abstract}

Passiflora L. (Passifloraceae), commonly known as sour passion fruit, yellow passion fruit or granadilla belongs to the family Passifloraceae and consists of approximately 465 species distributed all over the world except in Arctic and Antarctic regions. The present investigation focused on the foliar morphological diversity of the widely variant genus, Passiflora, at the inter and intra specific levels, with a view to understand the significance of foliar features in the systematics of the genus. The study was undertaken in 27 accessions including 8 species, 5 varieties and 2 hybrids of Passiflora, for analyzing both the qualitative and quantitative characteristics of leaves and trichomes. The investigation revealed the significance of foliar characters in the delimitation of the taxa at the inter specific and intra specific levels in the genus. Diversity at species level was noticed in characters like shape and size of leaf margin, pattern of leaf lobing, size and shape of stipules, number of umbilical glands, length of petiole, type of stomata, and glandular and non glandular trichomes. However, intra specific diversity was evident from the nature of leaf lobing, and in the distribution of glandular and non glandular trichomes. A taxonomic key based on the foliar features was constructed in support of the systematics of Passiflora.

Copy Right, IJAR, 2016,. All rights reserved.

\section{Introduction:-}

Passiflora L., the largest genus of the family Passifloraceae consists of about 465 species under 24 subgenera [1-2]. According to Feuillet and McDougal [3], Passiflora is a monophyletic group distributed in the subtropical and tropical regions of the world. The genus is distinguished by the herbaceous or woody climbers with characteristic three to five angled stem. Majority of the species possesses nectar secreting foliar and bracteole glands. Moreover, a wide range of diversity was observed in the foliar features of the genus.

Review of related literature revealed that the studies undertaken focused mainly on genetic diversity analysis [4-5] and cytological characterization [6]. Morphological and molecular variability in the wild species of Passiflora was

Corresponding Author:- VL Beena.

Address:- Research Scholar, Department of Botany, University of Kerala, Kariavattom, Thiruvananthapuram, 
reported by Viana et al. [7]. Krosnick [8] suggested the role of floral morphology in discriminating the species of Passiflora. However, investigations on the foliar characteristics is sparse except that of Tangarife et al. [9] who reported foliar variations in a few species of Passiflora from Columbia. The significance of leaf morphological and epidermal characters in plant systematics has been proved in Alternanthera Frpsk.(Amaranthacae)[10], Cassia sensu (Cesalpineaceae) [11], Senna (Fabaceae) [12] and Cassiinae (Leguminosae) and in Hybanthus (Violaceae) [13]. The present investigation makes use of foliar features to evaluate the role of those characters in the delimitation of the genus at the inter and intra specific levels.

\section{Materials and Methods:-}

Leaf morphological and trichome characteristics of 27 taxa of Passiflora belonging to eight species, five varieties and two hybrids collected from different parts of the states of Kerala and Tamil Nadu were analysed for the study. The plants were maintained in the Botanical Garden, Department of Botany, University of Kerala for the investigation. Details of the accessions and the places of collection are provided in Table 1. Both the qualitative (28) and quantitative (11) characters were analyzed and each character state had been scored (Table 2). Fifth leaf of the flowering branch was examined for the study to ensure uniform treatment, and the mean value of 10 observations were analysed. The data were subjected to Analysis of Variance (ANOVA) at the level of significance $\mathrm{p}<0.05$, and was used to test the significant differences among the taxa. Discriminant function analyses such as cluster analysis and scatter plot were carried out to check out the character differences among the taxa. Morphology of foliar trichomes and glands were examined with the help of Stereo microscopic (Olympus SZ) observations and a taxonomic key was prepared based on the findings.

\section{Result and Analysis:-}

Details of the qualitative characters analysed in the 27 taxa of eight species are are given in Table 3 . Foliar variations at the inter specific and intra specific levels were evident from the qualitative data analyzed (Table $3 \&$ Fig 1). Analysis of Variance performed on quantitative traits (Table 2) showed significant variations $(\mathrm{P}<0.05)$ at the inter and intra specific levels. Mean standard deviation, range, and coefficient of variation and $\mathrm{F}$ values of the quantitative traits were also undertaken (Table 3). A range of values were found as the average means of lamina length $(6.03 \mathrm{~cm}$ to $6.63 \mathrm{~cm})$, lamina width $(4.88 \mathrm{~cm}$ to $5.38 \mathrm{~cm})$, petiole length $(2.23 \mathrm{~cm}$ to $2.88 \mathrm{~cm})$, frequency of stomata in the upper and lower epidermis $(74.92 \mu \mathrm{m}$ to $78.70 \mu \mathrm{m}$ and $33.76 \mu \mathrm{m}$ to $36.44 \mu \mathrm{m})$, length and width of stomatal complex $(16.97 \mu \mathrm{m}$ to $17.82 \mu \mathrm{m} \& 13.42 \mu \mathrm{m}$ to $13.68 \mu \mathrm{m})$, length and width of guard cells $(18.14 \mu \mathrm{m}$ $18.56 \mu \mathrm{m} \& 5.09 \mu \mathrm{m}-5.55 \mu \mathrm{m})$, length and width of stoma $(9.77 \mu \mathrm{m}-9.97 \mu \mathrm{m} \& 3.21 \mu \mathrm{m}-3.98 \mu \mathrm{m})$. Among these, laminar width (LMW) showed maximum coefficient of variation (38.3372\%), whereas $F$ value (155.274) and width of guard cell (WGD) had minimum coefficient of variation (16.12\%) and F value (14.773).

The Principal Component Analysis (PCA) revealed that the characters were distributed among three principal axes (Fig 2) which accounted for approximately $99.672 \%$ of the total variance. The first principal component with Eigen value 17.432 explained $64.563 \%$ variance (Table 4). It was found that leaf lobing, leaf arrangement, petiole grove, leaf texture, leaf size, leaf shape, leaf margin, marginal sheath spacing, order of marginal sheath, lob tip gland, leaf color, tooth apex, sinus shape, leaf tip, leaf base, nature of hair, venation, stomata, and leaf pubescence were the highest loaded traits and had positive values. The second component accounted for $13 \%$ of the variability with dominance of characters like leaf lobing, leaf arrangement, petiole grove, leaf texture, leaf size, leaf color, leaf shape, leaf margin, order of margin sheath, marginal sheath spacing, tooth apex, sinus shape, leaf tip, leaf base, lob tip gland, nature of hair, and venation. The third principal component accounted for $6 \%$ of the variability with dominance of characters like leaf lobing, leaf color, leaf shape, leaf margin, order of margin sheath, leaf tip, leaf trichome and lobe tip gland. The most loaded characters that accounted for more variability in PC1, PC2 and PC3 included leaf lobing, leaf color, leaf shape, leaf margin, marginal sheath spacing, order of margin sheath, sinus shape, leaf tip, leaf trichome, lob tip gland and leaf pubescence, which distinguished the different taxa of the genus Passiflora at the inter and intra specific levels.

UPGMA dendrogram based on morphological differentiation of the accessions (Fig 3) revealed two principal clusters at an Euclidian distance 1.40. The first principal cluster consisted of P.coccinia, P.vitifolia, P.trifaciata and P.subpeltata along with two accessions of P.edulis var. edulis (acc4 \& acc5), whereas the second cluster possessed two sub clusters. All the varieties of P.foetida were grouped together in the second sub cluster along with the species, P.apoda and P.quadrangularis. However close relationship among the accessions of P.foetida var.foetida (acc 7, acc 8, acc 9, acc 12, acc 13 and acc14) was evident even though the accessions 10 and 11 differed 
extensively from the others. It was observed that the accession $P$. foetida var. gossippifolia was a connecting link between $P$. foetida var.foetida and $P$. foetida var.hispida. The two hybrid varieties of Passiflora (P. 'manapany' and $P$. 'canelle'), were clustered together with the varieties of P.edulis var. flavicarpa and P.edulis var. panamared, but the accessions of P.edulis var.edulis (acc 4 and acc 5) were seen grouped in the sub cluster of the second principal cluster.

A taxonomic key constructed based on the foliar features and the trichome characteristics distinguished the taxa at the inter and intra specific levels.

Table 1:- Accessions of Passiflora and the places of collection.

\begin{tabular}{|c|c|c|c|c|c|c|c|}
\hline Subgenera & $\begin{array}{l}\text { Sectio } \\
n\end{array}$ & Series & Species & Variety & $\begin{array}{l}\text { No.Acce } \\
\text { ssion }\end{array}$ & $\begin{array}{l}\text { Place of } \\
\text { collection }\end{array}$ & $\begin{array}{l}\text { Altitud } \\
\mathrm{e} \\
(\mathrm{ft})\end{array}$ \\
\hline \multirow[t]{2}{*}{ Plectostema } & Cieca & & P.apoda & & 1 & TVM & 112 \\
\hline & $\begin{array}{l}\text { Decal } \\
\text { oba }\end{array}$ & Miserae & P.trifaciata & & 1 & TVM & 112 \\
\hline \multirow[t]{3}{*}{ Distephna } & & & $\begin{array}{l}\text { P.coccinea } \\
\text { P.vitifolia }\end{array}$ & & $\begin{array}{l}1 \\
1\end{array}$ & $\begin{array}{l}\text { TVM } \\
\text { TVM }\end{array}$ & $\begin{array}{l}112 \\
112\end{array}$ \\
\hline & & Quadrangulareae & P.quadrangularis & & 1 & KKD & 75 \\
\hline & & Lobatae & P.subpeltata & & 1 & KTM & 59 \\
\hline \multirow[t]{4}{*}{$\begin{array}{l}\text { Granadilla } \\
\text { (Passiflora) }\end{array}$} & & & P.edulis & edulis & 3 & $\begin{array}{l}\text { KVTM, } \\
\text { KLM, } \\
\text { MUNR }\end{array}$ & $\begin{array}{l}112 \\
35 \\
2956 \\
\end{array}$ \\
\hline & & Incarnatae & P.edulis & flaviocarpa & 2 & $\begin{array}{l}\text { MUNR } \\
\text { EDKI }\end{array}$ & $\begin{array}{l}2956 \\
2565\end{array}$ \\
\hline & & & P.edulis & panamared & 1 & TVM & 112 \\
\hline & & & P.foetida & foetida & 8 & $\begin{array}{l}\text { MTPLY } \\
\text { M } \\
\text { CHTA } \\
\text { MDRI, } \\
\text { TSSR, } \\
\text { KULPZA } \\
\text { PTTA,V } \\
\text { M }\end{array}$ & $\begin{array}{l}171 \\
181 \\
175 \\
29 \\
106 \\
70 \\
112\end{array}$ \\
\hline \multirow{2}{*}{ Dysosmia } & & & P.foetida & hispida & 4 & $\begin{array}{l}\text { KLM, } \\
\text { TSSR, } \\
\text { KULPZA } \\
\text { KVTM }\end{array}$ & $\begin{array}{l}35 \\
29 \\
106 \\
112\end{array}$ \\
\hline & & & P.foetida & gossipifolia & 1 & TVM & 112 \\
\hline
\end{tabular}

*TVM Trivandrum, KVTM Kariyavattom, KLM Kollam, KKD Kozhikkodu, EDKI Edukki, MTPLYM

Mettuppalayam, CHTA Chenkotta, TSSR Thrissur, KULPZA Kulathuppuzha, ,MUNR Munnar, PTTA

Pathanamthitta, MDRI Madhurai 
Table 2:- List of qualitative and quantitative characters analysed with their character states Qualitative character.

\begin{tabular}{|c|c|c|}
\hline No & character & Description \\
\hline 3 & Leaf arrangement & '0' opposite; '1' alternate; '2' whorled \\
\hline 5 & Petiole grove & '0' present; '1' absent \\
\hline 6 & Leaf texture & '0’ glabrous; '1' pubescent; '2' fleshy \\
\hline 9 & Leaf shape & '0' ovate; '1' hastate; '2' pinnately trilobed; '3' palmately trilobed \\
\hline 10 & Leaf margin & $\begin{array}{l}\text { '0' serrate with gland; ' } 1 \text { ' serrate without gland; ' } 2 \text { ' entire with gland; ' } 3 \text { ' } \\
\text { entire without gland; ' } 4 \text { ' crenate with gland; ' } 5 \text { ' crenate without gland; ' } 6 \text { ' } \\
\text { ciliate; ' } 7 \text { ' wavy serrate with gland; ' } 8 \text { ' wavy serrate without gland }\end{array}$ \\
\hline 11 & Marginal teeth order & '0' $1^{\text {st }}$ order; ' 1 ' $2^{\text {nd }}$ order; ' 2 ' $3^{\text {rd }}$ order \\
\hline 15 & Sinus shape & '0' angular; ' 1 ' rounded \\
\hline 16 & Leaf tip & '0' acute; '1'accuminate; '2' cuspidate; '3' mucronate; '4' obtuse \\
\hline 17 & Leaf base & '0' attenuate; '1' shortly attenuate; '2' cordate; '3' subcordate; '4' hastate \\
\hline 18 & Nature of hair & '0' hispid; '1' puberlose; '2' hirsuite; '3' peltate; '4' stellate; '5' glandular \\
\hline 19 & Venation & '0' pinnately reticulate; ' 1 ' palmately reticulate \\
\hline 20 & Oil gland & '0' present; ' 1 ' absent \\
\hline 21 & Umbellical gland & '0' present; '1' absent \\
\hline 22 & Umbellical gland position & $\begin{array}{l}\text { ' } 0 \text { ' near to the laminar junction; ' } 1 \text { ' away from the laminar junction; ' } 2 \text { ' } \\
\text { throughout the petiole }\end{array}$ \\
\hline 23 & Lobe tip gland & '0' absent; '1' present \\
\hline 1 & Laminar length $(\mathrm{cm})$ & LML \\
\hline 2 & Laminar width area $(\mathrm{cm})$ & LMW \\
\hline 3 & Length of guard cell $(\mu \mathrm{m})$ & LGD \\
\hline 4 & Width of guard cell $(\mu \mathrm{m})$ & WGD \\
\hline 5 & Length of stomatal complex $(\mu \mathrm{m})$ & LSC \\
\hline 6 & Width of stomatal complex $(\mu \mathrm{m})$ & WSC \\
\hline 7 & Length of stomata $(\mu \mathrm{m})$ & LSto \\
\hline 8 & Width of stomata $(\mu \mathrm{m})$ & WSto \\
\hline 9 & Lower stomatal density(mm) & LSD \\
\hline 10 & Upper stomatal density (mm) & USD \\
\hline
\end{tabular}


Table 3:- Inter and intra specific qualitative data.

\begin{tabular}{|c|c|c|c|c|c|c|c|c|c|c|c|c|c|c|}
\hline Accession & $\begin{array}{c}\text { Leaf } \\
\text { lobi } \\
\text { ngt }\end{array}$ & $\begin{array}{l}\text { Stip } \\
\text { ule }\end{array}$ & $\begin{array}{c}\text { Leaf } \\
\text { Arrange } \\
\text { ment }\end{array}$ & $\begin{array}{c}\text { Peti } \\
\text { ole } \\
\text { Nat } \\
\text { ure }\end{array}$ & $\begin{array}{c}\text { Peti } \\
\text { ole } \\
\text { Gro } \\
\text { ve }\end{array}$ & $\begin{array}{l}\text { Leaf } \\
\text { text } \\
\text { ure }\end{array}$ & $\begin{array}{c}\text { Le } \\
\text { af } \\
\text { col } \\
\text { or }\end{array}$ & $\begin{array}{c}\text { Le } \\
\text { af } \\
\text { siz } \\
\text { e }\end{array}$ & $\begin{array}{c}\text { Lea } \\
f \\
\text { sha } \\
\text { pe }\end{array}$ & $\begin{array}{c}\text { Leaf } \\
\text { mar } \\
\text { gin }\end{array}$ & $\begin{array}{c}\text { Margi } \\
\text { nal } \\
\text { teeth } \\
\text { order }\end{array}$ & $\begin{array}{c}\text { Margi } \\
\text { nal } \\
\text { teeth } \\
\text { spaci } \\
\text { ng }\end{array}$ & $\begin{array}{c}\text { Tee } \\
\text { th } \\
\text { sha } \\
\text { pe }\end{array}$ & $\begin{array}{c}\text { Tee } \\
\text { th } \\
\text { ape } \\
x\end{array}$ \\
\hline $\begin{array}{l}\text { P. edulis } \\
\text { var. } \\
\text { flavicarpa }\end{array}$ & 0 & 0 & 1 & 0 & 0 & 0 & 1 & 0 & 3 & 0 & 0 & 1 & 0 & 1 \\
\hline $\begin{array}{l}\text { P. edulis } \\
\text { var. } \\
\text { flavicarpa }\end{array}$ & 0 & 0 & 1 & 0 & 0 & 0 & 1 & 0 & 3 & 0 & 0 & 1 & 0 & 1 \\
\hline $\begin{array}{l}\text { P. edulis } \\
\text { var. } \\
\text { flavicarpa }\end{array}$ & 0 & 0 & 1 & 0 & 0 & 0 & 1 & 0 & 3 & 0 & 0 & 1 & 0 & 1 \\
\hline $\begin{array}{l}\text { P. edulis } \\
\text { var.edulis }\end{array}$ & 0 & 0 & 1 & 0 & 0 & 0 & 2 & 0 & 3 & 2 & 0 & 1 & 0 & 1 \\
\hline $\begin{array}{l}\text { P. edulis } \\
\text { var edulis }\end{array}$ & 0 & 0 & 1 & 0 & 0 & 0 & 2 & 0 & 3 & 2 & 0 & 1 & 0 & 1 \\
\hline $\begin{array}{l}\text { P. edulis } \\
\text { panamared }\end{array}$ & 0 & 0 & 1 & 0 & 0 & 0 & 2 & 0 & 3 & 2 & 0 & 1 & 0 & 0 \\
\hline $\begin{array}{l}\text { P. foeitida } \\
\text { var.foeitida }\end{array}$ & 0 & 1 & 1 & 0 & 0 & 1 & 0 & 1 & 3 & 6 & 0 & 1 & 2 & 4 \\
\hline $\begin{array}{l}P . \text { foeitida } \\
\text { var.foeitida }\end{array}$ & 0 & 1 & 1 & 0 & 0 & 1 & 0 & 1 & 3 & 6 & 0 & 1 & 2 & 4 \\
\hline $\begin{array}{l}P . \text { foeitida } \\
\text { var.foeitida }\end{array}$ & 0 & 1 & 1 & 0 & 0 & 1 & 0 & 1 & 3 & 6 & 0 & 1 & 2 & 4 \\
\hline $\begin{array}{l}P . \text { foetida } \\
\text { var.feitida }\end{array}$ & 0 & 1 & 1 & 0 & 0 & 1 & 0 & 1 & 3 & 6 & 0 & 1 & 2 & 4 \\
\hline $\begin{array}{l}P . \text { foetida } \\
\text { var.foetida }\end{array}$ & 0 & 1 & 1 & 0 & 0 & 1 & 0 & 1 & 3 & 6 & 0 & 1 & 2 & 4 \\
\hline $\begin{array}{l}P . \text { foetida } \\
\text { var.foetida }\end{array}$ & 0 & 1 & 1 & 0 & 0 & 1 & 0 & 1 & 3 & 6 & 0 & 1 & 2 & 4 \\
\hline $\begin{array}{l}\text { P.foetida } \\
\text { var.foetida }\end{array}$ & 0 & 1 & 1 & 0 & 0 & 1 & 0 & 1 & 3 & 6 & 0 & 1 & 2 & 4 \\
\hline $\begin{array}{l}P . \text { foetida } \\
\text { var foetida }\end{array}$ & 0 & 1 & 1 & 0 & 0 & 1 & 0 & 1 & 3 & 6 & 0 & 1 & 2 & 4 \\
\hline $\begin{array}{l}P . \text { foetida } \\
\text { var. hispida }\end{array}$ & 0 & 1 & 1 & 0 & 0 & 1 & 0 & 1 & 3 & 6 & 0 & 1 & 2 & 4 \\
\hline $\begin{array}{l}\text { P. foetida } \\
\text { var.hispida }\end{array}$ & 0 & 1 & 1 & 0 & 0 & 1 & 0 & 1 & 3 & 6 & 0 & 1 & 2 & 4 \\
\hline $\begin{array}{l}P . \text { foetida } \\
\text { var.hispida }\end{array}$ & 0 & 1 & 1 & 0 & 0 & 1 & 0 & 1 & 3 & 6 & 0 & 1 & 2 & 4 \\
\hline $\begin{array}{l}\text { P. foetida } \\
\text { var.hispida }\end{array}$ & 0 & 1 & 1 & 0 & 0 & 1 & 0 & 1 & 3 & 6 & 0 & 1 & 2 & 4 \\
\hline $\begin{array}{l}\text { P. foetida } \\
\text { var.gossipp } \\
\text { ifolia }\end{array}$ & 0 & 1 & 1 & 0 & 0 & 1 & 3 & 1 & 3 & 6 & 0 & 1 & 2 & 4 \\
\hline P. vitifolia & 0 & 2 & 1 & 0 & 0 & 1 & 3 & 0 & 3 & 7 & 0 & 1 & 0 & 4 \\
\hline P. coccinia & 1 & 2 & 1 & 0 & 0 & 1 & 4 & 0 & 0 & 7 & 0 & 1 & 0 & 0 \\
\hline P.trifaciata & 0 & 2 & 1 & 0 & 1 & 0 & 2 & 1 & 2 & 3 & 0 & 1 & 0 & 0 \\
\hline P. apoda & 0 & 2 & 1 & 0 & 0 & 1 & 1 & 1 & 2 & 3 & 0 & 1 & 0 & 0 \\
\hline $\begin{array}{l}\text { P.quadrang } \\
\text { ularis }\end{array}$ & 0 & 2 & 1 & 0 & 0 & 0 & 1 & 0 & 0 & 3 & 0 & 1 & 0 & 0 \\
\hline P.subpeltat & 0 & 2 & 1 & 0 & 1 & 0 & 1 & 1 & 2 & 3 & 0 & 1 & 0 & 0 \\
\hline
\end{tabular}




\begin{tabular}{|l|c|c|c|c|c|c|c|c|c|c|c|c|c|c|}
\hline a & & & & & & & & & & & & & & \\
\hline $\begin{array}{l}P . \text { 'Manapa } \\
\text { ny' }\end{array}$ & 0 & 2 & 1 & 0 & 0 & 0 & 3 & 1 & 2 & 7 & 0 & 1 & 0 & 0 \\
\hline $\begin{array}{l}P . \\
\text { 'Cannelle' }\end{array}$ & 0 & 2 & 1 & 0 & 0 & 1 & 1 & 1 & 2 & 7 & 0 & 1 & 0 & 0 \\
\hline
\end{tabular}

'0'absent '1'present '2'small '3'medium '4' large ‘8'dense '9'sparse

Continued..........

\begin{tabular}{|c|c|c|c|c|c|c|c|c|c|c|c|c|c|c|}
\hline Accession & $\begin{array}{l}\text { Sin } \\
\text { us } \\
\text { sha } \\
\text { pe }\end{array}$ & $\begin{array}{l}\text { Le } \\
\text { af } \\
\text { tip }\end{array}$ & $\begin{array}{l}\text { Le } \\
\text { af } \\
\text { ba } \\
\text { se }\end{array}$ & $\begin{array}{c}\text { Nat } \\
\text { ure } \\
\text { of } \\
\text { hair }\end{array}$ & $\begin{array}{l}\text { Vena } \\
\text { tion }\end{array}$ & $\begin{array}{l}\text { Oil } \\
\text { gla } \\
\text { nd }\end{array}$ & $\begin{array}{l}\text { Umbe } \\
\text { llical } \\
\text { gland }\end{array}$ & $\begin{array}{l}\text { Umbe } \\
\text { llical } \\
\text { gland } \\
\text { positi } \\
\text { on }\end{array}$ & $\begin{array}{l}\text { Lo } \\
\text { be } \\
\text { tip } \\
\text { gla } \\
\text { nd }\end{array}$ & $\begin{array}{l}\text { Sm } \\
\text { ell }\end{array}$ & $\begin{array}{c}\text { Pubes } \\
\text { cent }\end{array}$ & $\begin{array}{c}\text { Leaf } \\
\text { polymor } \\
\text { phism }\end{array}$ & $\begin{array}{l}\text { Trich } \\
\text { ome }\end{array}$ & $\begin{array}{c}\text { Sto } \\
\text { mata }\end{array}$ \\
\hline $\begin{array}{l}\text { P. edulis } \\
\text { var. } \\
\text { flavicarpa }\end{array}$ & 0 & 0 & 2 & 0 & 1 & 1 & 0 & 0 & 1 & 1 & 0 & 1 & 0 & 1 \\
\hline $\begin{array}{l}\text { P. edulis } \\
\text { var. } \\
\text { flavicarpa }\end{array}$ & 0 & 0 & 2 & 0 & 1 & 1 & 0 & 0 & 1 & 1 & 0 & 1 & 0 & 1 \\
\hline $\begin{array}{l}\text { P. edulis } \\
\text { var. } \\
\text { flavicarpa }\end{array}$ & 0 & 0 & 2 & 0 & 1 & 1 & 0 & 0 & 1 & 1 & 0 & 1 & 0 & 1 \\
\hline $\begin{array}{l}\text { P. edulis } \\
\text { var.edulis }\end{array}$ & 0 & 0 & 2 & 0 & 1 & 1 & 0 & 0 & 1 & 1 & 0 & 1 & 0 & 1 \\
\hline $\begin{array}{l}\text { P. edulis } \\
\text { var.edulis }\end{array}$ & 0 & 0 & 2 & 0 & 1 & 1 & 0 & 0 & 1 & 1 & 0 & 1 & 0 & 1 \\
\hline $\begin{array}{l}P . \text { edulis } \\
\text { panamare } \\
d\end{array}$ & 0 & 0 & 2 & 0 & 1 & 1 & 0 & 0 & 1 & 1 & 0 & 1 & 0 & 1 \\
\hline $\begin{array}{l}\text { P. foeitida } \\
\text { var.foeitid } \\
a\end{array}$ & 1 & 0 & 2 & 5 & 1 & 0 & 0 & 2 & 1 & 2 & 9 & 1 & 9 & 1 \\
\hline $\begin{array}{l}\text { P. foeitida } \\
\text { var.foeitid } \\
a\end{array}$ & 1 & 0 & 2 & 5 & 1 & 0 & 0 & 2 & 1 & 2 & 9 & 1 & 9 & 1 \\
\hline $\begin{array}{l}\text { foeitida } \\
\text { var.foeitid } \\
a\end{array}$ & 1 & 0 & 2 & 5 & 1 & 0 & 0 & 2 & 1 & 2 & 9 & 1 & 9 & 1 \\
\hline $\begin{array}{l}\text { P. foeitida } \\
\text { var.foeitid } \\
a\end{array}$ & 1 & 0 & 2 & 5 & 1 & 0 & 0 & 2 & 1 & 2 & 9 & 1 & 9 & 0 \\
\hline $\begin{array}{l}\text { P. foeitida } \\
\text { var.foeitid } \\
a\end{array}$ & 1 & 0 & 2 & 5 & 1 & 0 & 0 & 2 & 1 & 2 & 9 & 1 & 9 & 0 \\
\hline $\begin{array}{l}\text { P. foeitida } \\
\text { var.foeitid } \\
\text { a }\end{array}$ & 1 & 0 & 2 & 5 & 1 & 0 & 0 & 2 & 1 & 2 & 8 & 1 & 8 & 1 \\
\hline $\begin{array}{l}\text { 3P.foeitid } \\
a \\
\text { var.foeitid } \\
a\end{array}$ & 1 & 0 & 2 & 5 & 1 & 0 & 0 & 2 & 1 & 2 & 8 & 1 & 8 & 1 \\
\hline $\begin{array}{l}\text { P. foeitida } \\
\text { varfoeitid } \\
\text { a }\end{array}$ & 1 & 0 & 2 & 5 & 1 & 0 & 0 & 2 & 1 & 2 & 8 & 1 & 8 & 1 \\
\hline P. foeitida & 1 & 0 & 2 & 2 & 1 & 0 & 0 & 2 & 1 & 2 & 8 & 1 & 8 & 1 \\
\hline
\end{tabular}




\begin{tabular}{|c|c|c|c|c|c|c|c|c|c|c|c|c|c|c|}
\hline varhispida & & & & & & & & & & & & & & \\
\hline $\begin{array}{l}\text { P. foeitida } \\
\text { var.hispid } \\
a\end{array}$ & 1 & 0 & 2 & 2 & 1 & 0 & 0 & 2 & 1 & 2 & 8 & 1 & 8 & 1 \\
\hline $\begin{array}{l}\text { P. foeitida } \\
\text { var.hispid } \\
a\end{array}$ & 1 & 0 & 2 & 2 & 1 & 0 & 0 & 2 & 1 & 2 & 9 & 1 & 9 & 1 \\
\hline $\begin{array}{l}\text { P. foeitida } \\
\text { var.hispid } \\
a\end{array}$ & 1 & 0 & 2 & 2 & 1 & 0 & 0 & 2 & 1 & 1 & 9 & 1 & 9 & 1 \\
\hline $\begin{array}{l}P . \text { foeitida } \\
\text { var.gossip } \\
\text { pifolia }\end{array}$ & 1 & 0 & 2 & 0 & 1 & 0 & 0 & 2 & 1 & 1 & 9 & 0 & 9 & 1 \\
\hline P. vitifolia & 1 & 0 & 2 & 1 & 1 & 0 & 0 & 0 & 1 & 1 & 1 & 1 & 1 & 1 \\
\hline $\begin{array}{l}P . \\
\text { coccinia }\end{array}$ & 0 & 0 & 3 & 1 & 1 & 0 & 0 & 0 & 1 & 1 & 1 & 1 & 1 & 1 \\
\hline $\begin{array}{l}\text { P.trifaciat } \\
a\end{array}$ & 0 & 4 & 2 & 0 & 1 & 1 & 0 & 0 & 0 & 1 & 0 & 1 & 0 & 1 \\
\hline P. apoda & 2 & 3 & 2 & 1 & 1 & 0 & 0 & 1 & 1 & 4 & 1 & 1 & 1 & 1 \\
\hline $\begin{array}{l}\text { P.quadran } \\
\text { gularis }\end{array}$ & 0 & 0 & 1 & 0 & 1 & 1 & 0 & 1 & 0 & 4 & 0 & 1 & 0 & 1 \\
\hline $\begin{array}{l}\text { P.subpelta } \\
\text { ta }\end{array}$ & 0 & 4 & 2 & 0 & 1 & 1 & 0 & 0 & 0 & 1 & 0 & 1 & 0 & 1 \\
\hline $\begin{array}{l}\text { P. 'Manap } \\
\text { any' }\end{array}$ & 0 & 0 & 2 & 0 & 1 & 1 & 0 & 0 & 1 & 1 & 0 & 1 & 0 & 1 \\
\hline $\begin{array}{l}P . \\
\text { 'Cannelle' }\end{array}$ & 1 & 0 & 2 & 1 & 1 & 0 & 0 & 0 & 1 & 2 & 1 & 1 & 1 & 1 \\
\hline
\end{tabular}

'0'absent '1'present '2'small '3'medium '4' large '8'dense '9'sparse

Table 4:- Mean, standard deviation, range, coefficient of variation and $F$ values of the quantitative traits.

\begin{tabular}{|c|l|l|l|l|l|}
\hline Character & Mean & SD & Range & CV (\%) & F value \\
\hline PTL & 15.945 & 1.467 & $6.03-6.63$ & 19.195 & $86.51^{* *}$ \\
\hline LML & 65.041 & 1.88 & $4.88-5.38$ & 30.2507 & $93.056^{* *}$ \\
\hline LMW & $\mathbf{4 1 . 8 2 5}$ & $\mathbf{2 4 . 4 2}$ & $\mathbf{2 . 2 3 - 2 . 8 8}$ & $\mathbf{3 8 . 3 3 3}$ & $\mathbf{1 5 5 . 5 7 4 * *}$ \\
\hline LGD & 1.57 & 1.122 & $1.40-7.70$ & 18.2055 & $26.835^{* *}$ \\
\hline WGD & $\mathbf{0 . 1 2}$ & $\mathbf{1 . 5 1}$ & $\mathbf{3 3 . 7 6 - 3 6 . 4 4}$ & $\mathbf{1 6 . 1 2 2 1}$ & $\mathbf{1 4 . 7 7 3 * *}$ \\
\hline LSC & 32.44 & 7.87 & $14.2-17.6$ & 20.7707 & $50.305^{* *}$ \\
\hline WSC & 98.063 & 1.43 & $2.0-10$ & 29.2374 & $28.096^{* *}$ \\
\hline LSto & 0.142 & 0.6147 & $2-5.10$ & 18.5206 & $47.951^{* *}$ \\
\hline W Sto & 1.4266 & .2768 & $.80-2.60$ & 19.4028 & $21.272^{* *}$ \\
\hline LSD & 1.8769 & 0.4066 & $.90-3.90$ & 21.6634 & $37.825^{* *}$ \\
\hline USD & 2.22 & 0.8164 & $1-6.70$ & 36.7748 & $47.47 * *$ \\
\hline
\end{tabular}

Table 5:- Principal component analysis in 27 taxa of Passiflora species accounted by the first three principal components.

\begin{tabular}{|l|c|c|c|}
\hline Variables & PC1 & PC2 & PC3 \\
\hline Leaf lobing & $\mathbf{0 . 3 3 2}$ & $\mathbf{- 0 . 5 0 4}$ & $\mathbf{0 . 8 5 0}$ \\
\hline Stipule & 0.092 & -0.191 & -0.025 \\
\hline Leaf arrangement & $\mathbf{- 0 . 2 9 1}$ & $\mathbf{0 . 3 1 2}$ & -0.081 \\
\hline Petiole nature & 0.092 & -0.191 & -0.060 \\
\hline petiole grove & $\mathbf{- 0 . 3 5 8}$ & $\mathbf{0 . 3 8 3}$ & -0.015 \\
\hline leaf texture & $\mathbf{- 0 . 4 8 8}$ & $\mathbf{- 0 . 2 9 3}$ & 0.072 \\
\hline leaf color & -0.933 & 0.34 & $\mathbf{0 . 2 8 8}$ \\
\hline leaf size & $\mathbf{- 0 . 8 4 2}$ & $\mathbf{- 0 . 6 8 9}$ & -0.127 \\
\hline
\end{tabular}




\begin{tabular}{|l|c|c|c|}
\hline leaf shape & $\mathbf{- 0 . 3 1 9}$ & $\mathbf{0 . 3 6 2}$ & $\mathbf{- 0 . 2 2 6}$ \\
\hline leaf margin & $\mathbf{0 . 3 3 2}$ & $\mathbf{- 0 . 5 0 4}$ & $\mathbf{0 . 8 5 0}$ \\
\hline order margin sheath & 0.847 & $\mathbf{- 0 . 3 8 7}$ & $\mathbf{0 . 7 1 4}$ \\
\hline Marginal sheath spacing & $\mathbf{0 . 6 5 4}$ & $\mathbf{0 . 2 8 0}$ & 0.265 \\
\hline Tooth shape & $\mathbf{- 0 . 2 1 6}$ & $\mathbf{- 0 . 2 8 0}$ & -0.114 \\
\hline sinus shape & $\mathbf{1 . 6 9 0}$ & $\mathbf{1 . 0 2 1}$ & -0.333 \\
\hline tooth apex & -0.078 & $\mathbf{- 0 . 3 8 8}$ & -0.015 \\
\hline leaf tip & $\mathbf{2 . 3 5 1}$ & $\mathbf{0 . 4 3 1}$ & $\mathbf{0 . 2 6 7}$ \\
\hline leaf base & $\mathbf{0 . 4 9 4}$ & $\mathbf{- 0 . 3 5 5}$ & -0.259 \\
\hline nature of hair & $\mathbf{0 . 8 2 3}$ & $\mathbf{0 . 7 2 2}$ & 0.233 \\
\hline Venation & $\mathbf{0 . 5 9 5}$ & -0.456 & -0.132 \\
\hline Stomata & $\mathbf{0 . 6 4 1}$ & -0.083 & -0.117 \\
\hline Presents of oil gland & -1.158 & -0.144 & -0.067 \\
\hline Umbilical gland position & -1.133 & -0.144 & 0.130 \\
\hline Pubescence lower leaf & $\mathbf{- 0 . 3 1 9}$ & $\mathbf{- 0 . 2 1 0}$ & $\mathbf{- 0 . 3 2 1}$ \\
\hline lob tip gland & -1.082 & $\mathbf{- 0 . 3 0 8}$ & $\mathbf{0 . 2 7 5}$ \\
\hline leaf trichome & $\mathbf{4 . 6 3 7}$ & $\mathbf{0 . 4 1 2}$ & -0.284 \\
\hline Eigen values & 17.432 & 3.664 & 1.764 \\
\hline Percent variation & 64.563 & 13.570 & 6.532 \\
\hline Cumulative percentage & 64.563 & 78.133 & 84.665 \\
\hline
\end{tabular}

\section{Discussion:-}

The analysis of variance on the quantitative data revealed significant differences among the taxa at $\mathrm{P}<0.05$ level. The highest coefficient of variation (Table 3.) estimated for laminar width $(38.3 \mathrm{~cm})$, upper stomatal density (36.7), laminar length $(30.25 \mathrm{~cm})$, and width of stomatal complex (29.23) suggests high degree of variability with regard to these characters. Of those, characters like laminar width and upper and lower stomatal density were found to be useful for distinguishing the taxa at inter specific level. Passiflora foetida var. gossippifolia was characterized by the presence of stomata on the adaxial surface, whereas in P.foetida var. foetida and P.foetida var.hispida they were amphistomatic. The lowest coefficient of variation was observed in characters like length and width of stomata and guard cells in varieties of $P$. foetida indicating that they had only a minimal role in delimiting the taxa at the intra specific level. The significance of stomata and trichomes in the delimitation of the varieties of P.edulis was reported earlier by Chrochemore et al. [3].

Principal component analysis using qualitative morphological data exhibited significant differences at the inter and intra specific levels among the wild and cultivated species of Passiflora (Table 4). Anlaysis of data revealed that characters like leaf lobing (0.850), leaf trichome(4.637), sinus shape (1.021), and leaf margin (0.850) were highly significant in differentiating the wild and cultivated taxa of Passiflora at the inter and intra specific levels. This may be the reason for the variation observed among the varieties of P.foetida. Researches like Viscosi and Cardini [14], Al-shammary and Gornall [15] and Ogundipe [10] emphasized the significance of foliar characters like lobing, leaf trichome, leaf margin and sinus shape in distinguishing the intra specific variations in legumes, members of Saxifragaceae and in Alternanthera respectively.

UPGMA dendrogram (Fig.2) revealed the close relationships between the two species, P. vitifolia and P.coccinea. This may be due to presence of multicellular trichomes with characteristic leaf lobing, leaf base, leaf color, and leaf shape and teeth apex. The presence of the two accessions of the P. edulis var. edulis (acc 5 and acc 4) in the first cluster and $P$. edulis var.flavicarpa and var. panamared in the second cluster suggest the significance of foliar morphology in the delimitation of the taxa at the varietal level. The three varieties were clearly demarcated by the color of the leaves and the nature of leaf margin. Even though inter varietal differentiation was noticed in the varieties of $P$. foetida, the foliar characters did not support the delimitation of varieties at the intra varietal level. However, separation of the accessions (acc10 and acc 11) of P.foetida var.foetida, from the rest of accessions may be due to the presence of anisocytic stomata and dense pubescent leaves. The clustering of the accessions of P.foetida var.hispida, into two different groups may be due to variations in the number and length of trichomes. The interlinking of P.foetida var.gossippifoia and the varieties P.foetida var.hispida and P.foetida var.foetida may be due to the foliar characters (absence of leaf polymorphism, trilobed leaves, lob tip gland and entire leaf margin with glands) shared by them. 
The variations in the foliar morphological characters at the inter specific levels were also noticed in the present investigation. Similarity in the entire leaf margin and the lack of trichomes and hairs in P.subpeltata and P.faciata pointed out the interspecific relationship. The presence of dense pubescence and multicellular trichomes in P.vitifolia and P.coccinea revealed their close relatioships. Simple leaves with entire margin in P.apoda and P. quadrangularis can be the distinguishing character to delimit the species. Occurrence of the two hybrid varieties P. 'Manapany' and P. 'Cananelle' together in the second subcluster may be due to the characters like leaf arrangement, leaf size, marginal sheath spacing and teeth apex shared by them, but the former was distinct by the presence of trichomes. According to Tangarife et al. [9] clustering of the seven species of Passiflora L. together in a single cluster based on the unique nature of the leaf arrangement and leaf margin.

The data suggest that the inter and intra specific variations in Passiflora may be primarily due to the foliar variations. The study emphasizes the significance of foliar characters in the delimitation of taxa at the inter and intra specific levels. The taxonomic key prepared, based on foliar features supports the findings.

\section{$P C$ values indicated in boldface are significant}

Fig 1:- Foliar morphological variation.
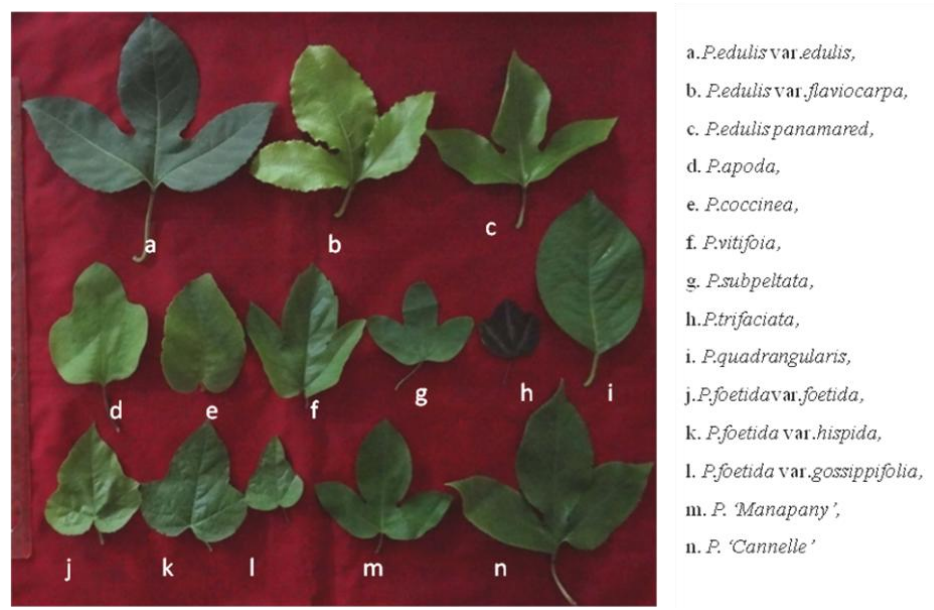

Fig 2:- PCA scatter plot exhibiting foliar variations in species of Passiflora.

\section{PCA variable loadings}

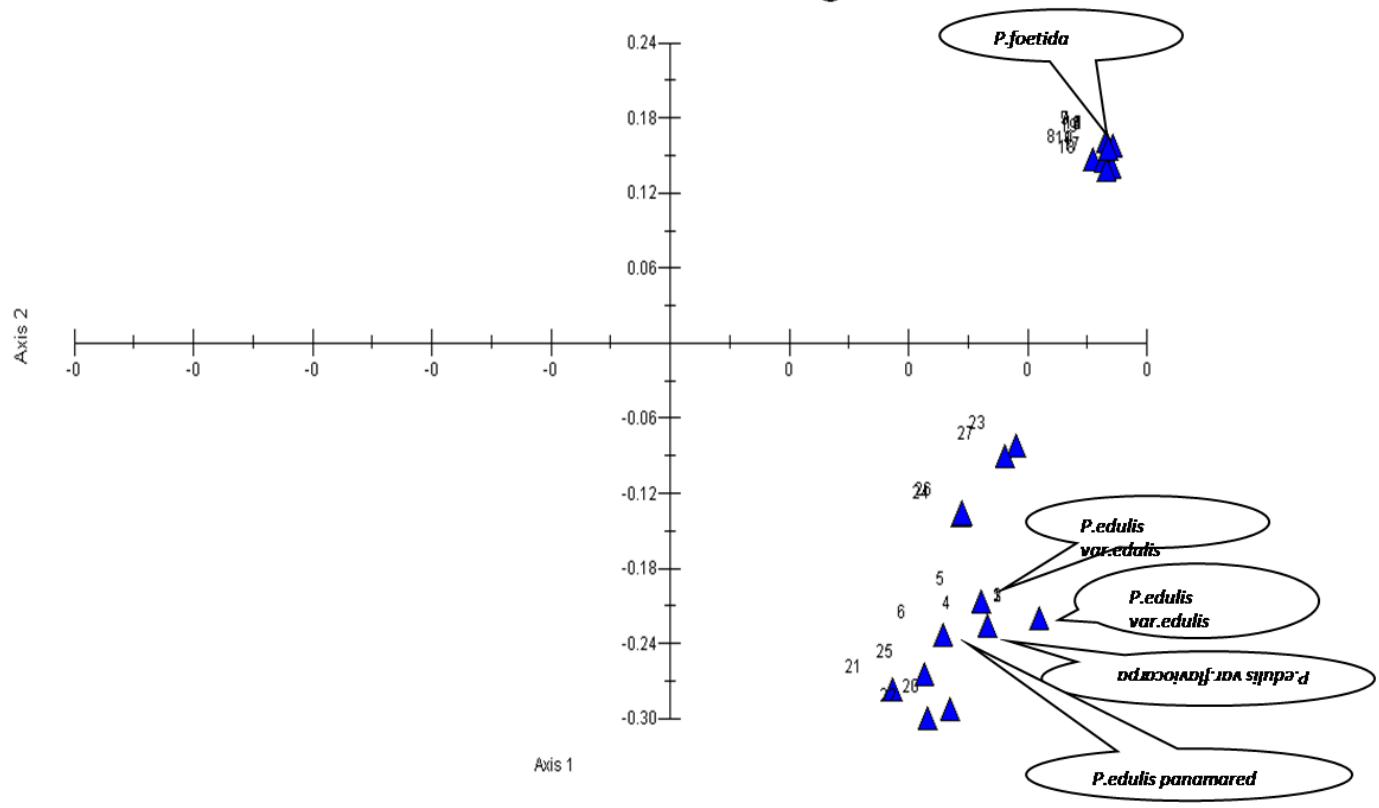


Fig 3:- Dendrogram based on UPGMA analysis generated from Euclidean distance of Leaf morphological characters.

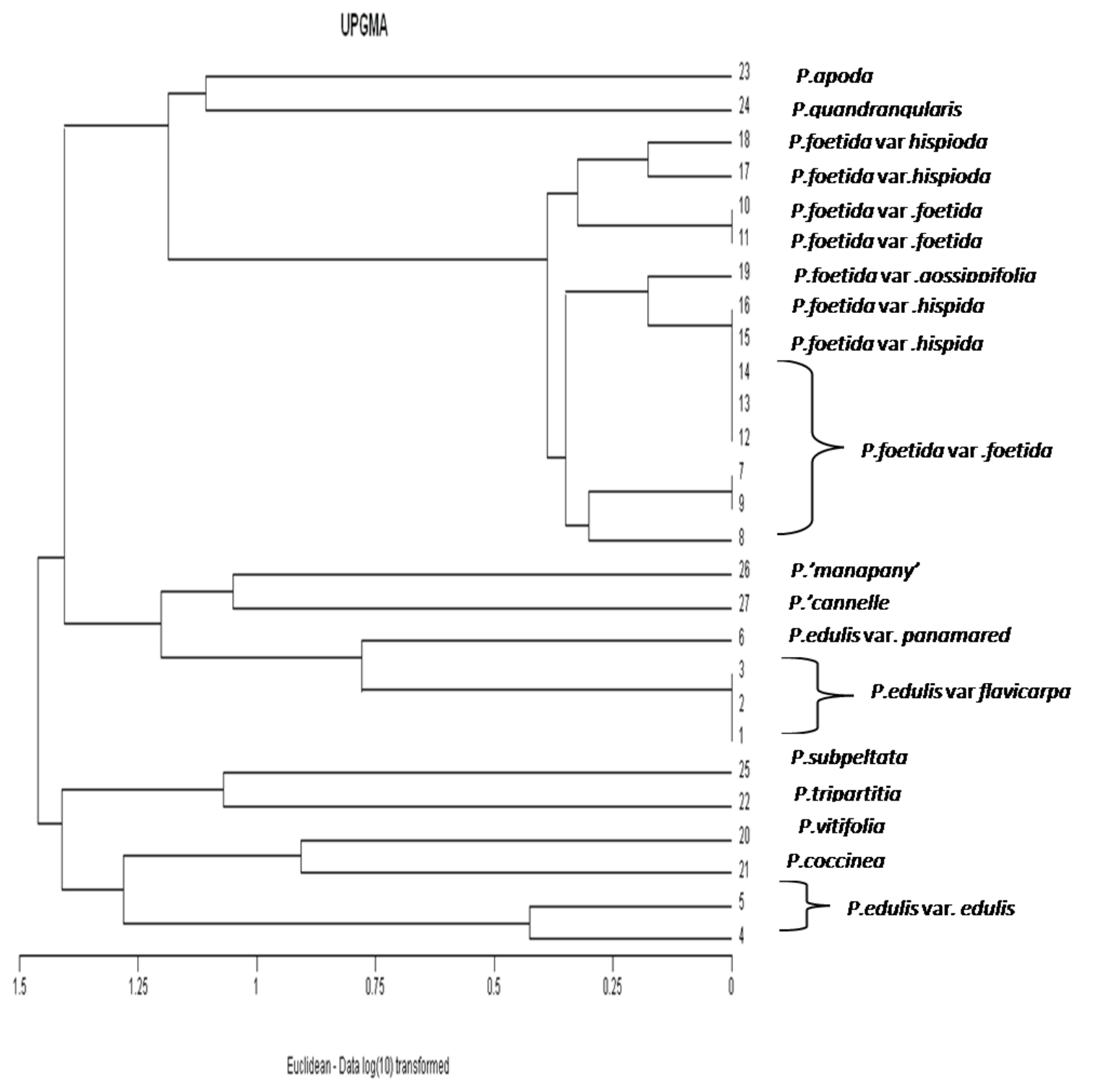

Taxonomic Key

Leaves simple

1. Glabrous with entire margin.........P.quadrangularis

2. Pubescent with serrate margin..............P. coccinea

Leaves lobed

1. Partially lobed, entire margin

2. Gland absent at the leaf tip

3. Leaves purple colored ..........P.trifaciata

4. Leaves green colored..............subpeltata

Gland present at the leaf tip

1. Trichomes are non glandular and multicellular ...P. apoda

2. Trichomes are both glandular and non glandular

3. Unicellular non glandular, dense hispid trichomes 
...P.foetida var. hispida

1. Unicellular non glandular, dense, pubescent trichomes

.P.foetida var. foetida

1. Unicellular non glandular, sparsely pubescent trichome,

Petiole with pink color

P.foetida var.gossippifolia

1. Deeply lobed, serrate margin

2. Glabrous leaves

3. Marginal gland present

4. Wavy serrate margin

5. Pale green color..................edulis var. edulis

6. Linear serrate margin

7. Dark green color

8. Green color petiole....P.edulis var flavicarpa

9. Red color petiole......... P.edulis 'panamared ‘

10. Marginal gland absent

11. Linear serrate margin dark green

color.......... 'Cannelle'

12. Leaves pubescent

13. Serrate margin, absent marginal gland, light green color P. 'Manapany'

\section{Acknowledgements:-}

The authors are grateful to Dr.Suhara beevy S, Head, Department of Botany, University of Kerala for providing the facilities.

\section{Reference:-}

1. Killip, E.P. (1938). The American species of Passifloraceae.- Field Museum of Natural History, Botanical Series - 19: 1-613.

2. Vanderplank, J. (1996). Passion flowers. MIT Press, United Kingdom.

3. Feuillet, C.Y., Mcdougal, J. (2003). A new infrageneric classification of Passiflora.- Passiflora 14(1), 1-4.

4. Crochemore, M.L., Molinari, H.B., Vieira, L.G.E. (2003). Genetic diversity in passion fruit (Passiflora spp.) evaluated by RAPD markers, Braz. Arch. Biol. Technol. 46, 521-527.

5. dos Santos, L.F., de Oliveira, E.J., dos Santos , de Carvalho, A.F.M., Costa, J.L., Padua, J.G. (2011). ISSR markers as a tool for the assessment of genetic diversity in Passiflora, Biochen genet. 49(7), 540-544.

6. Souza, M.M., Pereira, T.N.S., Vieira, M.L.C. (2008). Cytogenetic studies in some species of Passiflora L. (Passifloraceae): A review emphasizing Brazilian species, Braz.arch. boil. Technol. 51 247-258.

7. Viana, A.J.C., Souza, M.M., Araújo, I.S., Corrêa, R.X., Ahnert D. (2010). Genetic diversity in Passiflora species determined by morphological and molecular characteristics, Biologia Plantarum. 54 (3), 535-538.

8. Krosnick, S.E., Freadenstein, J.V. (2005). Monophyly and floral character homology of old world Passiflora (subgenus Decaloba:super section Disemma). Systematic botany 30(1), 139-152

9. Tangarife, M.M.M., Caetano, C.M., Tique, C.A.P. (2009).Morphological Characterization of colombian Passiflora species, ACTA Agromonica. 58 (3).

10. Ogundipe, O.T. (1996) Taxonomic significance of Leaf epidermis in Alternanthera Frpsk.(Amaranthacae), Bol.Soc.Brot.Ser. 2 ,231-244.

11. Petchsri, S., Boonkerd, T. (2003).Numerical taxonomy of Cassia sensu lato in Thailand. In BRT research reports 2003, Chuan printing press Ltd. Part., Bangkok

12. Soladoye, M.O. ,. Onakoya, M.A, Chukwuma, E.C.,. Sonibare, M.A (2010). Morphometric study of the genus Senna Mill.In South-western Nigeria, Afr. J. Plant Sci. 4, 044-052.

13. Saheed, S.A., Illoh, H.C. (2011). Important morphological characters in several species of Cassiinae (Leguminosae) in south-western Nigeria, Not. Sci. Biol. 3, 47-56

14. Viscosi, V., Cardini, A. (2011). Leaf Morphology, Taxonomy and Geometric Morphometrics: A Simplified Protocol for Beginners. PLoS ONE 6(10)

15. Al-shammary, K.I.A., Gornall, R.J. (1994). Trichome Anatomy of Saxifragaceae s.l. from the Southern Hemisphere, Botanical Journal of the Linnean Society.114, 99-131. 